\title{
Electrical tuning of the LC:PDMS channels
}

\author{
Katarzyna A. Rutkowska, ${ }^{* 1}$ Tomasz R. Woliński, ${ }^{1}$ Rita Asquini, ${ }^{2}$ Luca Civita, ${ }^{2}$ Luca Martini, ${ }^{2}$ \\ and Antonio d'Alessandro ${ }^{2}$ \\ ${ }^{1}$ Faculty of Physics, Warsaw University of Technology, Koszykowa 75, 00-662 Warszawa, POLAND \\ ${ }^{2}$ Department of Information Engineering, Electronics and Telecommunications, Sapienza University of Rome, \\ via Eudossiana 18, 00184 Roma, ITALY
}

Received March 21, 2017; accepted June 29, 2017; published June 30, 2017

\begin{abstract}
In this paper, studies are presented on electrical tuning of optical channels formed by the PDMS-based waveguides infiltrated with a liquid crystalline material. In particular, preliminary results are demonstrated of numerical simulations demonstrating changes in optical parameters of the waveguide channels when influenced by electric field applied via flat and IPS electrodes in order to fabricate photonic switches based on optical directional couplers. The first experimental trials of sputtering the ITO layer on PDMS substrate are also shown.
\end{abstract}

Polydimethylsiloxane (PDMS) [1], characterized by high optical transparency and low surface energy, electric constant, and Young's modulus, is widely recognized as an interesting, high-quality organic material for microand opto-fluidic functional systems [2, 3]. Another advantage of applying PDMS is its cheap and easy processing owing to the fact that PDMS-based photonic devices are typically fabricated with the use of reliable technology based on standard soft photolithography [2]. In this way, complex microstructures can be successfully manufactured in such flexible polymeric substrates at much lower costs when compared to traditional glass- or semiconductor-based components, commonly applied in integrated optical systems. Moreover, typical technological processing of PDMS, based on a casting and molding technique, allows for pattern and sophisticated geometries to be easily achieved. It gives an opportunity of shaping the waveguides with possible bends and intersections, which is particularly useful when creating functional elements for integrated optics such as e.g. $\mathrm{Y}$ - and $\mathrm{X}$-junctions, directional couplers and ring resonators. In principle, air-channels formed in PDMS can be additionally infiltrated with other liquid and gaseous materials. Specifically, what is particularly important in the analysed case, liquid crystals (LCs) may be successfully used as a guest (and moreover active) material to be introduced into microchannels formed in PDMS. In this context, LCs have been demonstrated as an effective medium to be applied as component elements (e.g. cores) in switchable and reconfigurable waveguides. In fact, this kind of photonic structures, taking advantage of high sensitivity to external fields and of nonlinear

\footnotetext{
*E-mail: kasia@if.pw.edu.pl
}

optical properties of LCs [4], may in turn result in the realization of novel, low cost and low energy consuming integrated photonic devices.

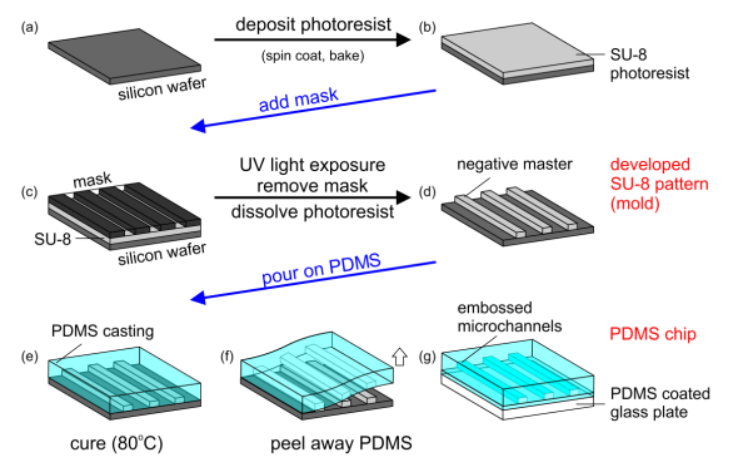

Fig. 1. Scheme of a molding and casting fabrication process leading to microchannels formation in a PDMS material.

Since the already mentioned casting and molding technique is the most popular fabrication process to obtain well-defined microstructures in a PDMS material, the same procedure may be applied to form LC:PDMS photonic devices under consideration. The formation of air microchannels to be infiltrated with a nematic liquid crystalline material is schematically shown in Fig. 1 and described in detail elsewhere [5-7]. In order to increase its efficiency, the infiltration process is performed in vacuum, at a temperature above the nematic LC (NLC) clearing point, i.e. in its isotropic phase. Then, the sample is cooled down to room temperature.
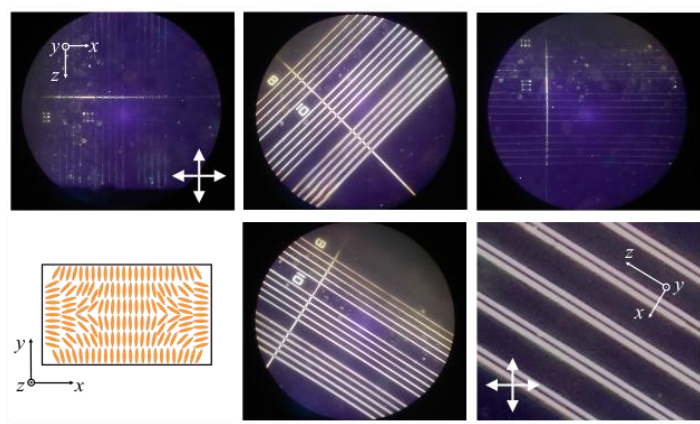

Fig. 2. Photos of a top view of PDMS waveguide channels infiltrated with LC observed under a polarized microscope while rotated with respect to crossed polarizers. The zoom of the waveguides channels 
when forming the angle of about $45^{\circ}$ with the polarizer axis is shown in the right-bottom corner, whereas the schematic drawing in the leftbottom corner represents waveguide cross-section and NLC molecular orientation inside.

The inspection of resultant LC:PDMS waveguides under a polarized microscope, as shown in Fig. 2, gives information about the molecular arrangement of the NLC inside PDMS channels. In fact, when analyzing a sequence of photos of the LC:PDMS waveguides observed between crossed polarizers, it is evident that NLC molecules are aligned hometropically with respect to each PDMS wall. This spontaneous molecular arrangement on the PDMS surface, appearing without any orienting layer, as observed previously by e.g. [8, 9], is obtained mainly due to the low energy surface of the PDMS, which forces a minimum contact interface between the PDMS surface and strongly elongated NLC molecules of a rod-like shape. Molecular orientation inside the waveguide channels, as presented e.g. in Fig. 3, has been found by assuming stiff homeotropic boundary conditions on PDMS walls and by performing numerical simulations with the use of a finite difference relaxation scheme. To be more precise, an alternative-direction successive over-relaxation (ADSOR) scheme [10] has been applied to find the solution of the Euler-Lagrange equation. The latter is in principle obtained as an effect of free energy minimization, while taking into account Frank's free energy of deformation and free energy related to molecular reorientation under the influence of external field(s) if applied [4]. Moreover, the multigrid method [10] has been applied together with the ADSOR scheme to make numerical simulations faster.

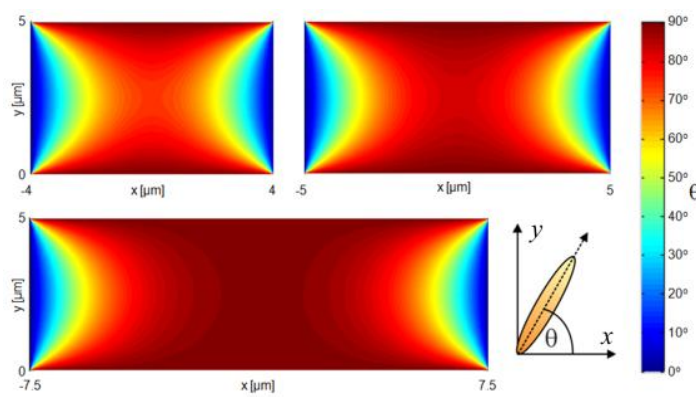

Fig. 3. Molecular arrangement inside the LC:PDMS waveguide channels of different widths $w=8,10$ and $15 \mu \mathrm{m}$ (with their crosssections shown in the graphs), as described by the spatial distribution of the orientation angle $\theta$ retrieved in numerical simulations.

With spatial distribution of the orientation angle $\theta$ retrieved in numerical simulations it is possible to find an effective refractive index spatial distribution within NLC cores for TE- and TM-like polarization in accordance to the following equation:

$$
n_{\mathrm{TM}, \mathrm{TE}}(\theta)=n_{o} n_{e}\left[\left(n_{e, o}^{2}-n_{o, e}^{2}\right) \cos ^{2} \theta+n_{o, e}^{2}\right]^{-1 / 2},
$$

where $n_{o}$ and $n_{e}$ are the ordinary and extraordinary refractive indices, respectively.
Taking above into consideration, it is easy to demonstrate that optical properties of LC:PDMS photonic structures can be relatively readily adjusted by changing the molecular orientation of NLC. As a possible solution one may consider an electric voltage application whose variation can be in principle used to tune light switching from one channel to another as eventually observed at the output of the LC:PDMS periodic structure.

To perform a proof-of-concept of such electrical tuning, two types of electrodes, namely flat and in-planeswitching (IPS), have been considered (see Fig. 5a). Moreover, two different liquid crystalline materials, namely E7 and 1110 (both synthesized at the Military University of Technology, Warsaw, Poland), have been chosen as the ones to be used for infiltration. Their refractive indices in comparison to that of a PDMS material (Sylgard 184 from Dow Corning) [11] are presented in Fig. 4. Please note that the 1110 NLC mixture is characterized by low birefringence $(\Delta \mathrm{n}=0.046$ ( $\lambda=589 \mathrm{~nm}$ and $20^{\circ} \mathrm{C}$ ) and negative electric anisotropy $(\Delta \varepsilon=-1.2)$ at $\mathrm{kHz}$ frequencies. Analogous values for $\mathrm{E} 7$ NLC are $\Delta \mathrm{n}=0.264$ and $\Delta \varepsilon=14.5$, respectively. The elastic constant taken into calculations (within one elastic constant approximation) is equal to $20 \mathrm{pN}$.

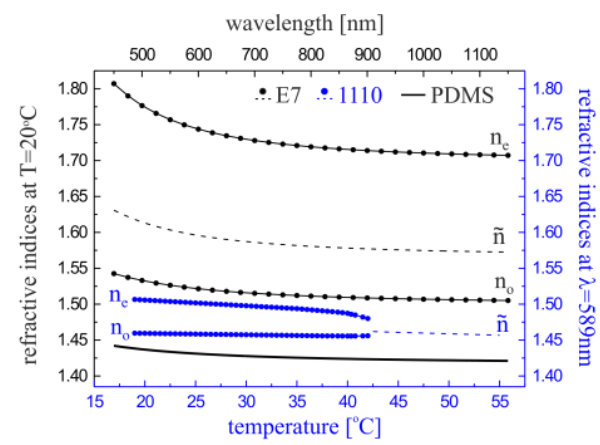

Fig. 4. Dispersion characteristics of E7 NLC and PDMS at room temperature (in black) and temperature dependence of refractive indices of 1110 NLC at a wavelength of $589 \mathrm{~nm}$ (in blue).

The same numerical scheme, i.e. ADSOR combined with the multigrid method (MM), has been applied to calculate molecular reorientation under the influence of an electric field. For this purpose the following steps are required: (i) calculation of electric potential for a given molecular orientation inside the waveguide channels and for specific electrodes geometry (ADSOR+MM); (ii) calculation of spatial electric field distribution in the region within the electrodes ( $E_{x}$ and $E_{y}$ components are simply calculated as the first derivatives of electric potential with respect to $x$ and $y$ direction; Fig. 5b); (iii) calculation of molecular orientation under the influence of the electric field applied (ADSOR+MM) applied for solve the suitable version of the Euler-Lagrange equation; Fig. 5c). Such a resultant molecular arrangement within NLC cores allows for calculation of 

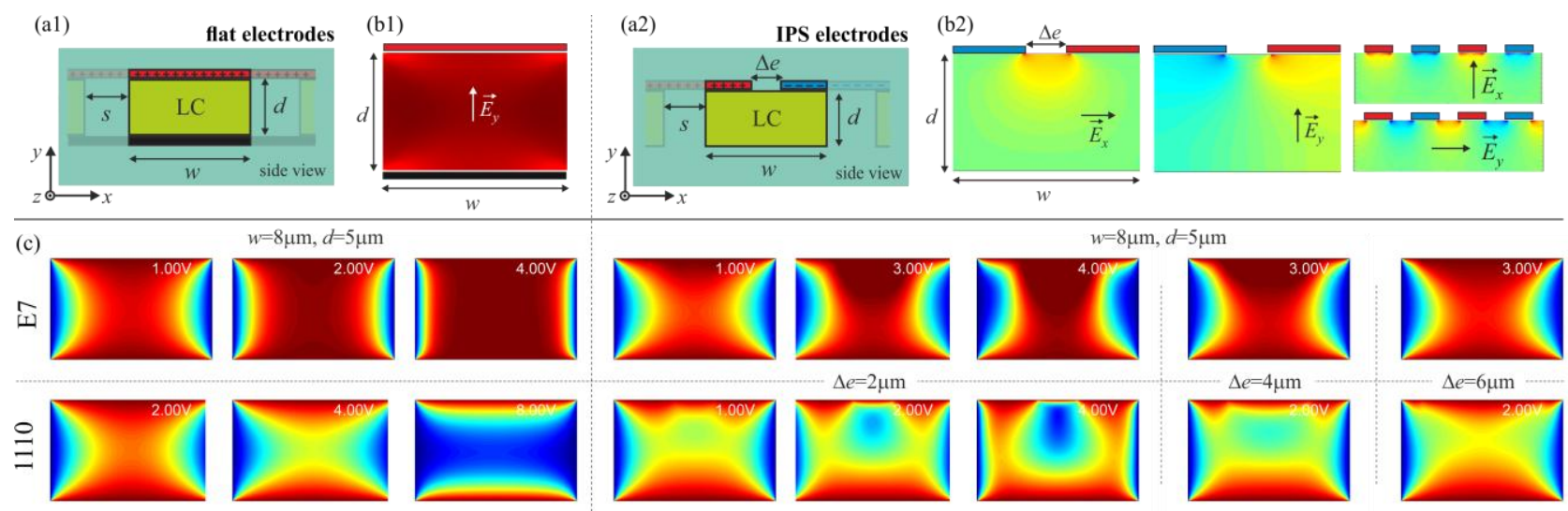

Fig. 5. Scheme of the electrodes (a1-a2) and the electric field distribution (b1-b2) calculated numerically for the spatial molecular orientation arrangement presented in Fig. 3. Graphs in a panel (c) demonstrates how the orientation angle is changing with the voltage for E7 and 1110 NLCs used for infiltrating the PDMS waveguide channels (the color-scale is the same as used in Fig. 2). In a case of IPS electrodes different gaps between electrodes have been analyzed.

the effective refractive index for TM- and TEpolarization, simply as a function of the orientation angle, as described by Eq. (1). Electro-optically controlled LC:PDMS channels have been used to design different optical directional couplers as basic structures to get optical switches. In addition, numerical simulations based on BPM [12, 13] have been performed for different geometrical parameters of the waveguides channels and electrodes, as well as for different wavelengths, voltages, polarization states and liquid crystalline materials. It has been concluded that the coupling lengths in LC:PDMS based directional couplers can be easily tuned, reaching a reasonable length of less than one millimeter, by changing the above mentioned variables varying the voltage to obtain direct and on-line tunability of the devices.
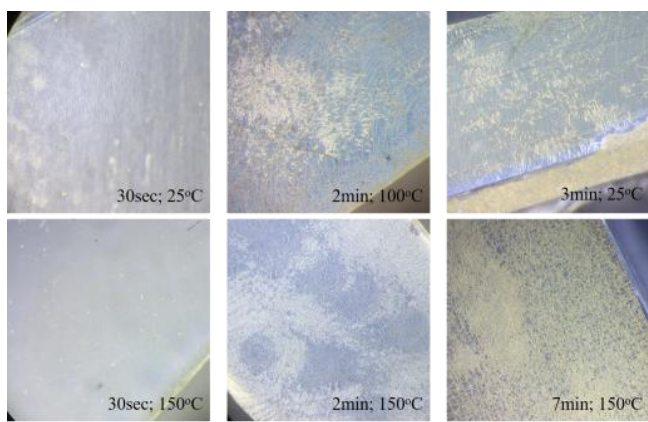

Fig. 6. Pictures of the PDMS surface after sputtering with ITO.

Deposition times and temperatures are given in each case.

In order to obtain the electro-optical control of the directional couplers analyzed here, flexible electrodes have been fabricated by sputtering ITO on the PDMS, with different deposition times of 30 s and more (up to several minutes), at the temperatures of $25^{\circ} \mathrm{C}, 100^{\circ} \mathrm{C}$ and $150^{\circ} \mathrm{C}$. As one can conclude from the photos shown in Fig. 6, improper sputtering parameters may result in cracking and wrinkling of the ITO on the PDMS surface. For the best depositions (e.g. $30 \mathrm{~s}$ at $150^{\circ} \mathrm{C}$ ), preliminary conductive ITO layers with a thickness of about $28 \mathrm{~nm}$ have been obtained.

In conclusion, the electric tuning of LC:PDMS couplers is feasible, as based on numerical simulations showing LC molecular reorientation due to the electric field applied, as well as on experimental demonstration of a preliminary conductive ITO layer sputter on the PDMS surface.

This work was partially supported by the COST Action IC 1208. K.A.R. would like to acknowledge the support from the Polish National Science Centre under the grant no. DEC-2013/11/B/ST7/04330.

\section{References}

[1] G. Wypych, Handbook of polymers (Elsevier 2016).

[2] J.C. McDonald, D.C. Duffy, J.R. Anderson, D.T. Chiu, H. Wu, O.J. Schueller, G.M. Whitesides, Electrophoresis 21, 27 (2000).

[3] Y. Fainman, L. Lee, D. Psaltis, C. Yang, Optofluidics: fundamentals, devices, and applications (McGraw-Hill, Inc., 2009).

[4] I. Khoo, Liquid crystals: physical properties and nonlinear optical phenomena (John Wiley \& Sons, 2007).

[5] A. d'Alessandro, L. Martini, L. Civita, R. Beccherelli, R. Asquini, Proc. SPIE 9384, 93840L (2015).

[6] R. Asquini, L. Martini, A. d'Alessandro, Mol. Cryst. Liquid Cryst. 614, 11 (2015).

[7] B. Bellini, J. F. Larchanché, J. P. Vilcot, D. Decoster, R. Beccherelli, A. d'Alessandro, Applied Optics 44, (33), (2005).

[8] L. De Sio, J.G. Cuennet, A.E. Vasdekis, D. Psaltis, Appl. Phys. Lett. 96, 131112 (2010)

[9] W. Zheng, L. Yang, M. Lee, Phot. Lett. Poland 3(1), 8 (2010).

[10] Y.Saad, Iterative methods for sparse linear systems (SIAM, 2003).

[11] F. Schneider, J. Draheim, R. Kamberger, U. Wallrabe, Sensors Actuat. A: Physical 151, 95 (2009).

[12] R. Asquini, A. d'Alessandro, Mol. Cryst. Liquid Cryst. 375, 243 (2002).

[13] R. Asquini, L. Civita, L. Martini, A. d'Alessandro, Mol. Cryst. Liquid Cryst. 619, 12 (2015) 